\title{
Non-Condensable Gases in RELAP-7
}

Joshua E Hansel, Matthias S Kunick, Ray A Berry, David Andrs

August 2018

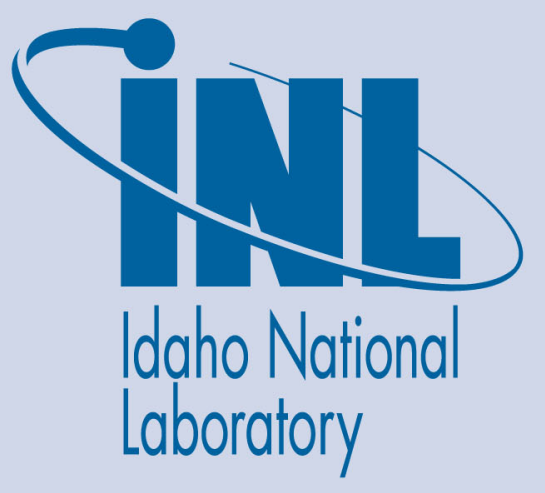

The INL is a U.S. Department of Energy National Laboratory operated by Battelle Energy Alliance 


\title{
Non-Condensable Gases in RELAP-7
}

\author{
Joshua E Hansel, Matthias S Kunick, Ray A Berry, David Andrs
}

August 2018

Idaho National Laboratory Idaho Falls, Idaho 83415

http://www.inl.gov

Prepared for the

U.S. Department of Energy

Under DOE Idaho Operations Office

Contract unknown 


\title{
Non-Condensable Gases in RELAP-7
}

\author{
Joshua E. Hansel ${ }^{1}$, Matthias Kunick ${ }^{1}$, Ray Berry ${ }^{1}$, and David Andrs ${ }^{1}$
}

${ }^{1}$ Idaho National Laboratory

August 2018

DOE Office of Nuclear Energy

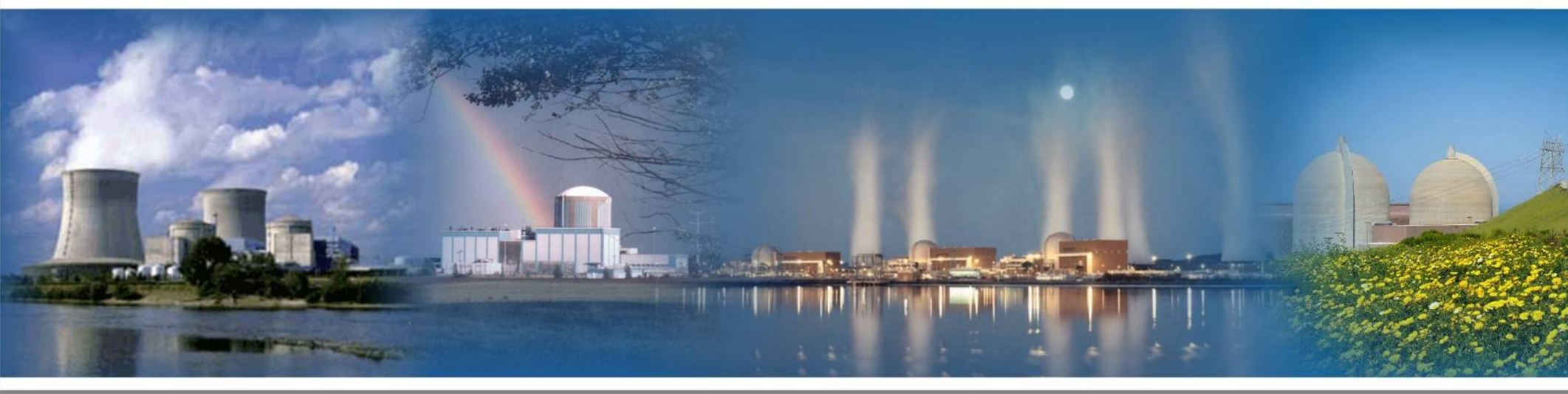




\title{
INL REPORT
}

INL/EXT-18-51163

Unlimited Release

Printed August 2018

\section{Non-Condensable Gases in RELAP-7}

\author{
Joshua E. Hansel ${ }^{1}$, Matthias Kunick ${ }^{1}$, Ray Berry ${ }^{1}$, and David Andrs ${ }^{1}$ \\ ${ }^{1}$ Idaho National Laboratory
}

Prepared by

Idaho National Laboratory

Idaho Falls, Idaho 83415

The Idaho National Laboratory is a multiprogram laboratory operated by

Battelle Energy Alliance for the United States Department of Energy

under DOE Idaho Operations Office. Contract DE-AC07-05ID14517.

Approved for public release; further dissemination unlimited.

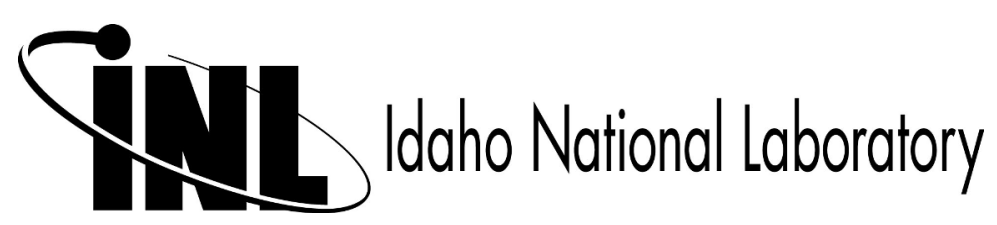


Issued by the Idaho National Laboratory, operated for the United States Department of Energy by Battelle Energy Alliance.

NOTICE: This report was prepared as an account of work sponsored by an agency of the United States Government. Neither the United States Government, nor any agency thereof, nor any of their employees, nor any of their contractors, subcontractors, or their employees, make any warranty, express or implied, or assume any legal liability or responsibility for the accuracy, completeness, or usefulness of any information, apparatus, product, or process disclosed, or represent that its use would not infringe privately owned rights. Reference herein to any specific commercial product, process, or service by trade name, trademark, manufacturer, or otherwise, does not necessarily constitute or imply its endorsement, recommendation, or favoring by the United States Government, any agency thereof, or any of their contractors or subcontractors. The views and opinions expressed herein do not necessarily state or reflect those of the United States Government, any agency thereof, or any of their contractors.

Printed in the United States of America. This report has been reproduced directly from the best available copy.

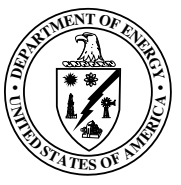


INL/EXT-18-51163

Unlimited Release

Printed August 2018

\section{Non-Condensable Gases in RELAP-7}

Joshua E. Hansel ${ }^{1}$, Matthias Kunick ${ }^{1}$, Ray Berry ${ }^{1}$, and David Andrs ${ }^{1}$

${ }^{1}$ Idaho National Laboratory 


\section{Contents}

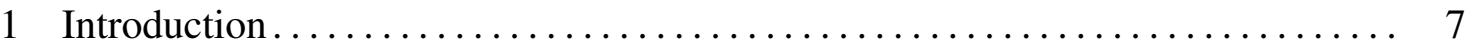

2 Mixture Models .......................................... 8

2.1 Mixture Model A - Components Under Partial Pressures ............ 8

$2.1 .1 \quad$ Calculation of density . . . . . . . . . . . . . . . . . . 10

2.1.2 Calculation of mass-specific properties ............... 10

2.1.3 Calculation of transport properties $\ldots \ldots \ldots \ldots \ldots \ldots \ldots \ldots .11$

2.2 Mixture Model B - Components Possess Partial Volumes ............. 11

2.2.1 Calculation of density . . . . . . . . . . . . . . . . . . 12

2.2.2 Calculation of mass-specific properties .............. 12

2.2.3 Calculation of transport properties $\ldots \ldots \ldots \ldots \ldots \ldots \ldots \ldots \ldots \ldots$

2.2.4 Calculations from given specific volume and internal energy ...... 13

2.3 Calculation of Heat Capacities and Speed of Sound from Thermodynamic Derivatives .................................. 14

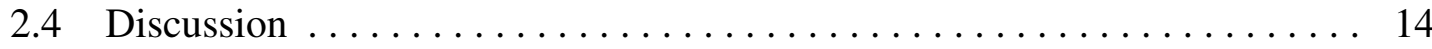

2.4.1 Equivalence of model A and B for ideal gases ............ 14

2.4.2 Inconsistency in model A for non-ideal gases............. 15

2.4.3 Potential pitfall of model B . . . . . . . . . . . . . . . . . . . 17

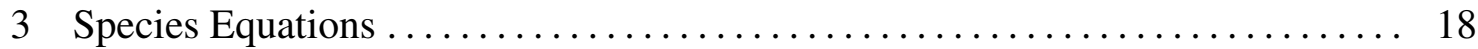

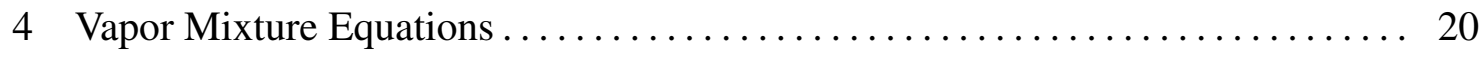

4.1 Spatial Discretization and Boundary Conditions ................ 21

References ............................................. 22 


\section{Figures}

1 Illustration of model A - Components under partial pressures possess mix-

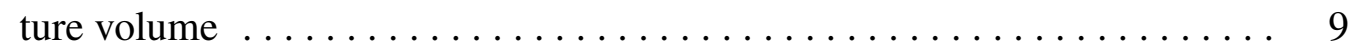

2 Illustration of model B - Components under pressure of the mixture possess partial volumes $\ldots \ldots \ldots \ldots \ldots \ldots \ldots \ldots \ldots \ldots \ldots \ldots \ldots \ldots$ 


\section{Introduction}

This report considers how the model PDEs in RELAP-7 can be amended to account for the presence of non-condensable gases (NCGs) in the flow system. Let the number of NCGs be denoted by $N_{\text {NCG }}$. The primary (and condensable) vapor, e.g., steam, will be referred to as the "primary vapor" and will have the subscript " $v, 0$ ", and the other constituents are assumed to be non-condensable and will be referred to as "NCGs" and will use the subscript " $v, i$ ", with $i=1, \ldots, N_{\mathrm{NCG}}$. Mixture quantities will use the subscript " $v$ " (without any numeric index).

Before distinguishing between condensable and non-condensable gases, the mixture equations are derived generally; there the mixture components are just referred to as "species" and will just have a subscript " $i$ " instead of the corresponding vapor subscript " $v, i$ ". The total number of species is denoted by $N_{\text {species. }}$.

This report is organized as follows: Section 2 discusses mixture models, Section 3 discusses the governing equations for transport of the mixture species, and Section 4 gives the system of equations to be used for the inclusion of NCGs. 


\section{Mixture Models}

State-of-the-art mixture models are formulated as functions of the dimensionless Helmholtz free energy

$$
\phi(\delta, \tau, \psi)=\phi^{\circ}(\delta, \tau, \psi)+\phi^{\mathrm{r}}(\delta, \tau, \psi),
$$

where the reducing parameters of the reduced density $\delta=\rho / \rho_{c}(\psi)$ and the inverse reduced temperature $\tau=T_{c}(\psi) / T$ are composition-dependent functions, $\phi^{\circ}$ is the ideal-gas part of the mixture, and $\phi^{\mathrm{r}}$ is the residual part of the non-ideal mixture. The ideal and residual parts are commonly written as

$$
\begin{gathered}
\phi^{\mathrm{o}}(\rho, T, \psi)=\sum_{i=1}^{N_{\text {species }}} \psi_{i}\left[\phi_{\mathrm{o} i}^{\mathrm{o}}(\rho, T)+\ln \psi_{i}\right], \\
\phi^{\mathrm{r}}(\delta, \tau, \psi)=\sum_{i=1}^{N_{\text {species }}} \psi_{i} \phi_{\mathrm{o} i}^{\mathrm{r}}(\delta, \tau)+\Delta \phi^{\mathrm{r}}(\delta, \tau, \psi) .
\end{gathered}
$$

In Eqs. (2) and (3), the subscript o indicates the pure component equation of state, and $\Delta \phi^{\mathrm{r}}$ is the empirical departure function that describes the difference in the residual Helmholtz free energy of the mixture with regard to that obtained from the linear combination of the residual Helmholtz free energies of the pure components. The state-of-the-art mixture model requires the reduced Helmholtz free energies of the pure components along with continuous first, second, and third derivatives. The calculation of phase equilibria and inverse functions is especially computationally expensive, and convergence cannot be guaranteed. For these reasons, simplified mixture models are often used in extensive process simulations. The resulting inaccuracies in the calculated fluid properties must be accepted.

In this section, two different models, A and B, for the ideal mixing of non-ideal gases are discussed. These models will turn out to be equivalent for ideal-gas mixtures but lead to different results when applied to non-ideal gases. These differences, along with potential pitfalls and possible enhancements, are discussed.

\subsection{Mixture Model A - Components Under Partial Pressures}

For this model it is assumed that all of the $N_{\text {species }}$ components are under their partial pressures $p_{i}$ and possess the entire volume $V$ of the mixture as illustrated in Figure 1. 


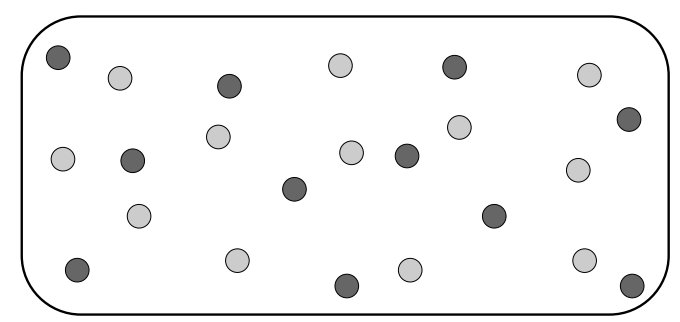

Figure 1. Illustration of model A - Components under partial pressures possess mixture volume

The partial pressures $p_{i}$ are defined in terms of the molar fractions $\psi_{i}$ as

$$
p_{i}=\psi_{i} p
$$

where the molar fraction $\psi_{i}$ is calculated from

$$
\psi_{i}=\frac{n_{i}}{n}
$$

with

$$
n=\sum_{i=1}^{N_{\text {species }}} n_{i} .
$$

The symbol $n$ refers to the amount of substance (number of moles). The mass fraction $\xi_{i}$ of each component is defined as

$$
\xi_{i}=\frac{m_{i}}{m}
$$

where the mass of the mixture $m$ is

$$
m=\sum_{i=1}^{N_{\text {species }}} m_{i} .
$$

The molar mass $M$ of the mixture is computed from

$$
M=\sum_{i=1}^{N_{\text {species }}}\left(\psi_{i} M_{i}\right)
$$

or from

$$
M=\frac{1}{\sum_{i=1}^{N_{\text {species }}} \frac{\xi_{i}}{M_{i}}} .
$$


The mass fraction $\xi_{i}$ can be obtained from the molar fraction using

$$
\xi_{i}=\frac{M_{i}}{M} \psi_{i}
$$

\subsubsection{Calculation of density}

If the molar fraction equals the volume fraction, the density $\rho=m / V$ can be written as

$$
\rho=\sum_{i=1}^{N_{\text {species }}} \frac{m_{i}}{V}=\sum_{i=1}^{N_{\text {species }}} \rho_{i}\left(p_{i}, T\right) .
$$

Eq. (12) with the partial densities computed form partial pressures only holds for ideal gas mixtures, where

$$
\rho=\sum_{i=1}^{N_{\text {species }}} \rho_{i}\left(p_{i}, T\right)=\sum_{i=1}^{N_{\text {species }}} \frac{p_{i} M_{i}}{\bar{R} T}=\frac{p}{\bar{R} T} \sum_{i=1}^{N_{\text {species }}} \psi_{i} M_{i}=\frac{p M}{\bar{R} T} .
$$

Eqs. (4) and (13) are known as Dalton's law, which only applies to ideal-gas mixtures as shown in Section 2.4. For non-ideal components, Eq. (12) should not be used. The use of Eq. (15) is recommended instead.

\subsubsection{Calculation of mass-specific properties}

All mass-specific properties $z$, including specific volume, should be calculated from

$$
z=\sum_{i=1}^{N_{\text {species }}} \frac{Z_{i}}{m}=\sum_{i=1}^{N_{\text {species }}} \frac{m_{i} z_{i}\left(p_{i}, T\right)}{m}=\sum_{i=1}^{N_{\text {species }}} \xi_{i} z_{i}\left(p_{i}, T\right) .
$$

For specific volume, Eq. (14) yields

$$
v=\sum_{i=1}^{N_{\text {species }}} \frac{V_{i}}{m}=\sum_{i=1}^{N_{\text {species }}} \frac{m_{i} v_{i}\left(p_{i}, T\right)}{m}=\sum_{i=1}^{N_{\text {species }}} \xi_{i} v_{i}\left(p_{i}, T\right) .
$$

For the ideal-gas mixture, this is consistent with Eqs. (12) and (13) since

$$
v=\sum_{i=1}^{N_{\text {species }}} \xi_{i} v_{i}\left(p_{i}, T\right)=\sum_{i=1}^{N_{\text {species }}} \xi_{i} \frac{\bar{R}, T}{p_{i} M_{i}}=\frac{\bar{R}, T}{p M} .
$$


This equation is more suitable for ideal mixtures of real gases since thermodynamic consistency of the mass-specific properties can be assured. For the specific entropy it must be noted that the entropy of mixing is already included when using Eq. (14).

\subsubsection{Calculation of transport properties}

All transport properties $z$, such as dynamic viscosity and thermal conductivity, should be calculated from

$$
z=\sum_{i=1}^{N_{\text {species }}} \psi_{i} z_{i}\left(p_{i}, T\right) .
$$

The volume fraction is approximated with the molar fraction, and it is assumed that the transport properties of the mixture depend on that volume fraction and the pure component transport properties only.

\subsection{Mixture Model B - Components Possess Partial Volumes}

For this model it is assumed that all of the $N_{\text {species }}$ components are under the pressure of the mixture $p$ and reside in a fraction of the total volume as illustrated in Figure 2.

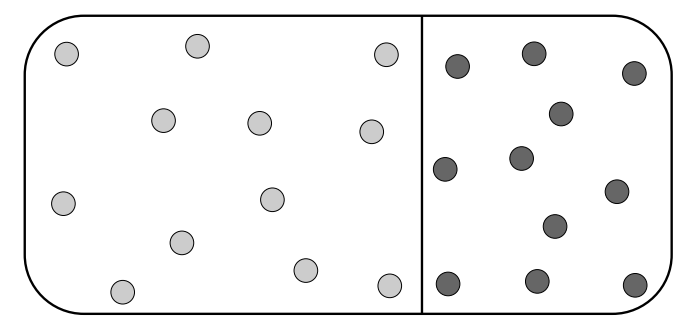

Figure 2. Illustration of model B - Components under pressure of the mixture possess partial volumes

The partial volumes $V_{i}$ are defined using the molar fractions $\psi_{i}$ as

$$
V_{i}=\psi_{i} V,
$$


where the molar fraction $\psi_{i}$ is calculated from Eq. (5) or, for given mass fractions, from

$$
\psi_{i}=\frac{M}{M_{i}} \xi_{i}
$$

with the molar mass $M$ of the mixture computed from Eq. (10).

\subsubsection{Calculation of density}

The specific volume of the mixture is defined as

$$
v(p, T)=\frac{V}{m}=\frac{\sum_{i=1}^{N_{\text {species }}} m_{i} v_{i}(p, T)}{m}=\sum_{i=1}^{N_{\text {species }}} \xi_{i} v_{i}(p, T) .
$$

The density is $\rho=1 / v$. It must be noted that the specific volume $v$ of component $i$ must not be confused with the partial specific volume, i.e., the inverse of the partial density in model A (see Eq. (12)). For model B, the partial volume of component $i$ must be calculated from

$$
v_{i}=\frac{v}{\xi_{i}}
$$

which is in agreement with the definition of the mass-fraction in Eq. (7).

\subsubsection{Calculation of mass-specific properties}

All mass-specific properties $z$, including specific volume, are calculated from

$$
z(p, T)=\sum_{i=1}^{N_{\text {species }}} \frac{Z_{i}}{m}=\sum_{i=1}^{N_{\text {species }}} \frac{m_{i} z_{i}(p, T)}{m}=\sum_{i=1}^{N_{\text {species }}} \xi_{i} z_{i}(p, T) .
$$

For the ideal-gas mixture, Eq. (20) is equal to Eq. (16) since

$$
v(p, T)=\sum_{i=1}^{N_{\text {species }}} \xi_{i} v_{i}(p, T)=\sum_{i=1}^{N_{\text {species }}} \xi_{i} \frac{\bar{R} T}{p M_{i}}=\sum_{i=1}^{N_{\text {species }}} \psi_{i} \frac{\bar{R} T}{p M}=\frac{\bar{R} T}{p M} .
$$


Eq. (22) assures thermodynamic consistency of the mass-specific properties for the ideal mixing of real gases. For the specific entropy $s$, the entropy of mixing must be added when using Eq. (22), so that

$$
s(p, T)=\sum_{i=1}^{N_{\text {species }}} \xi_{i} s(p, T)-\sum_{i=1}^{N_{\text {species }}} \psi_{i} \ln \psi_{i}
$$

where $R$ is the specific gas constant of the mixture $R=\bar{R} / M$, where $\bar{R}$ is the universal gas constant, which has a value of $8.3144598 \mathrm{~J} /(\mathrm{mol}-\mathrm{K})$.

\subsubsection{Calculation of transport properties}

All transport properties $z$, such as dynamic viscosity and thermal conductivity, should be calculated from

$$
z(p, T)=\sum_{i=1}^{N_{\text {species }}} \psi_{i} z_{i}(p, T) .
$$

The volume fraction is approximated with the molar fraction and it is assumed that the transport properties of the mixture depend on that volume fraction and the pure component transport properties only.

\subsubsection{Calculations from given specific volume and internal energy}

The independent variables of the mixture model, pressure $p$ and temperature $T$, are calculated by iteration from

$$
\left[\begin{array}{c}
p(v, e, \xi) \\
T(v, e, \xi)
\end{array}\right]=\left[\begin{array}{c}
p \\
T
\end{array}\right]:\left\{\begin{array}{l}
v=\sum_{i=1}^{N_{\text {species }}} \xi_{i} v_{i}(p, T) \\
e=\sum_{i=1}^{N_{\text {species }}} \xi_{i} e_{i}(p, T)
\end{array}\right.
$$




\subsection{Calculation of Heat Capacities and Speed of Sound from Ther- modynamic Derivatives}

For both models A and B, the heat capacities and speed of sound must be calculated according to their definitions from thermodynamic derivatives:

$$
\begin{gathered}
c_{p}=\left(\frac{\partial h}{\partial T}\right)_{p}, \\
c_{v}=\left(\frac{\partial e}{\partial T}\right)_{v}, \\
c=v \sqrt{-\left(\frac{\partial p}{\partial v}\right)_{s} .}
\end{gathered}
$$

Since the independent parameters of the mixture model are $p$ and $T$, the derivatives must be calculated from the following equations:

$$
\begin{gathered}
\left(\frac{\partial e}{\partial T}\right)_{v}=\left(\frac{\partial e}{\partial T}\right)_{p}-\left(\frac{\partial e}{\partial p}\right)_{T} \frac{\left(\frac{\partial v}{\partial T}\right)_{p}}{\left(\frac{\partial v}{\partial p}\right)_{T}}, \\
\left(\frac{\partial p}{\partial v}\right)_{s}=\left[\left(\frac{\partial v}{\partial p}\right)_{T}-\left(\frac{\partial v}{\partial T}\right)_{p} \frac{\left(\frac{\partial s}{\partial p}\right)_{T}}{\left(\frac{\partial s}{\partial T}\right)_{p}}\right]^{-1} .
\end{gathered}
$$

\subsection{Discussion}

\subsubsection{Equivalence of model $A$ and $B$ for ideal gases}

For ideal gases, the mixture models A and B are equivalent. This can easily be derived from the ideal-gas equation

$$
p=\rho \frac{\bar{R} T}{M},
$$


where $\bar{R}$ is the universal gas constant. According to Eq. (32), the partial density of component $i$ as an ideal gas reads

$$
\rho_{i}^{A}=\frac{m_{i}}{V}=p_{i} \frac{M_{i}}{\bar{R} T},
$$

for model A. Eq. (12), in conjunction with Eqs. (4) and (9), gives the density of the mixture:

$$
\rho^{A}=\sum_{i=1}^{N_{\text {species }}}\left(p_{i} \frac{M_{i}}{\bar{R} T}\right)=\frac{\sum_{i=1}^{N_{\text {species }}}\left(p_{i} M_{i}\right)}{\bar{R} T}=\frac{p \sum_{i=1}^{N_{\text {species }}}\left(\psi_{i} M_{i}\right)}{\bar{R} T}=\frac{p M}{\bar{R} T} .
$$

For model $\mathrm{B}$, the (molecular) density of the ideal gas for component $i$ is

$$
\rho_{i}^{B}=\frac{m_{i}}{V_{i}}=\frac{m_{i}}{\psi_{i} V}=\frac{\rho_{i}^{A}}{\psi_{i}}=p \frac{M_{i}}{\bar{R} T},
$$

and, according to Eq. (20), the density of the mixture is

$$
\rho^{B}=\frac{1}{\sum_{i=1}^{N_{\text {species }}}\left(\frac{\xi_{i}}{\rho_{i}(p, T)}\right)}=\frac{1}{\sum_{i=1}^{N_{\text {species }}} \xi_{i}\left(\frac{\bar{R} T}{p M_{i}}\right)}=\frac{1}{\frac{\bar{R} T}{p} \sum_{i=1}^{N_{\text {species }}}\left(\frac{\xi_{i}}{M_{i}}\right)},
$$

which finally gives

$$
\rho^{B}=\frac{1}{\frac{\bar{R} T}{p} \sum_{i=1}^{N_{\text {species }}}\left(\frac{\psi_{i}}{M}\right)}=\frac{p M}{\bar{R} T} .
$$

The equivalence of the models in terms of the resulting mixture density can be seen in Eqs. (34) and (37).

\subsubsection{Inconsistency in model A for non-ideal gases}

For real gases, the models $\mathrm{A}$ and $\mathrm{B}$ are not equivalent. It can be shown that model $\mathrm{A}$ implies an inconsistency when it is applied to real fluids. The pressure $p$ as a function of density $\rho$ and temperature $T$ is calculated from a Helmholtz free energy explicit equation of state from

$$
p=\rho \frac{\bar{R}}{M} T\left(1+\delta \phi_{\delta}^{\mathrm{r}}\right)
$$


where $\delta=\rho / \rho_{c}$ is the reduced density, $\tau=T_{c} / T$ is the inverse reduced temperature, and $\phi=f /(\bar{R} T)$ is the dimensionless Helmholtz free energy. The superscript $\mathrm{r}$ denotes the residual part, the subscript $\delta$ denotes the first derivative with respect to the reduced density, and the subscript $c$ denotes the critical point. For consistency, the definition of the mass fraction given in Eq. (7) must lead to Eq. (10). For a binary mixture with the components I and II, Eq. (7) can be rewritten as

$$
\xi_{\mathrm{I}}=\frac{\rho_{\mathrm{I}}}{\rho}=\frac{\rho_{\mathrm{I}}}{\rho_{\mathrm{I}}+\rho_{\mathrm{II}}} .
$$

For simplicity, Eq. (38) is rewritten for the partial density as a function of partial pressure and temperature

$$
\rho_{i}=\frac{p_{i} M_{i}}{\bar{R} T} \frac{1}{\left(1+\delta \phi_{\delta}^{\mathrm{r}}\right)}=\frac{p_{i} M_{i}}{\bar{R} T} X_{i}
$$

Inserting Eq. (40) in Eq. (39) gives

$$
\xi_{\mathrm{I}}=\frac{p_{\mathrm{I}} M_{\mathrm{I}} X_{\mathrm{I}}}{p_{\mathrm{I}} M_{\mathrm{I}} X_{\mathrm{I}}+p_{\mathrm{II}} M_{\mathrm{II}} X_{\mathrm{II}}} .
$$

Using Eq. (4) with Eq. (41) yields to

$$
\xi_{\mathrm{I}}=\frac{\psi_{\mathrm{I}} M_{\mathrm{I}}}{\psi_{\mathrm{I}} M_{\mathrm{I}}+\psi_{\mathrm{II}} M_{\mathrm{II}}+\psi_{\mathrm{II}} M_{\mathrm{II}}\left(\frac{X_{\mathrm{II}}}{X_{\mathrm{I}}}-1\right)}=\frac{\psi_{\mathrm{I}} M_{\mathrm{I}}}{M+\psi_{\mathrm{II}} M_{\mathrm{II}}\left(\frac{X_{\mathrm{II}}}{X_{\mathrm{I}}}-1\right)} .
$$

Eq. (42) only agrees with Eq. (7) if $X_{\mathrm{I}} / X_{\mathrm{II}}=1$. This condition is only fulfilled for ideal gases. Similarly, only the density of ideal-gas mixtures can be calculated with Eq. (12). For non-ideal gases the definition of the partial pressures does not lead to volume fractions that are equal to the molar fractions.

For model A, the following relation is assumed:

$$
V=m v(p, T, \boldsymbol{\psi})=m_{i} v_{i}\left(p_{i}, T\right) .
$$

It can be shown that Eq. (43) is always fulfilled for ideal-gas mixtures since

$$
V=m \frac{\bar{R} T}{p M}=m_{i} \frac{\bar{R} T}{p_{i} M_{i}} .
$$

is fulfilled with Eqs. (4), (7), and (11). For non-ideal gases this will not be the case.

Mixture model A is used by TRACE, where the components are considered to be ideal gases. For mixing non-ideal gases, model A is not fully consistent. Therefore, the application of this model is not planned for RELAP-7. 


\subsubsection{Potential pitfall of model B}

The fluid properties of the individual components of model B are evaluated at the pressure of the mixture. For mixtures with condensable components, this restricts the applicability of model B to temperatures above the highest saturation temperature in the considered pressure domain. For example, humid air is a mixture of water vapor and non-condensable gases. At ambient conditions, the water vapor properties of the unsaturated humid air need to be calculated at conditions, where pure water would be liquid. In this case, the lowest temperature where model $\mathrm{B}$ is applicable would be $T_{\mathrm{s}}(p=0.1 \mathrm{MPa}) \approx 373 \mathrm{~K}$. An extrapolation into the metastable vapor phase would allow for a minimum temperature $T_{\min }(p=0.1 \mathrm{MPa}) \approx 326 \mathrm{~K}$ at the vapor spinodal. 


\section{Species Equations}

Assuming that each species can have its own velocity, the 1-D mass conservation equation for species $i$ is as follows:

$$
\frac{\partial \xi_{i} \rho}{\partial t}+\frac{\partial \xi_{i} \rho u_{i}}{\partial x}=S_{i}
$$

where $S_{i}$ is the volumetric mass source of the species, if any.

Let the mixture velocity be defined as follows:

$$
u \equiv \sum_{i=1}^{N_{\text {species }}} \xi_{i} u_{i} .
$$

Summing Equation (45) over all species gives

$$
\frac{\partial \rho}{\partial t}+\frac{\partial \rho u}{\partial x}=\sum_{i=1}^{N_{\text {species }}} S_{i},
$$

which looks identical to the single-vapor mass conservation equation. However, it is undesirable to track velocities for each species, so now we attempt to eliminate $u_{i}$. First we define the diffusion velocity for each species:

$$
\hat{u}_{i} \equiv u_{i}-u \text {. }
$$

Inserting this definition into Equation (45) gives

$$
\frac{\partial \xi_{i} \rho}{\partial t}+\frac{\partial \xi_{i} \rho\left(\hat{u}_{i}+u\right)}{\partial x}=S_{i}
$$

Defining a diffusive flux,

$$
J_{i} \equiv \xi_{i} \rho \hat{u}_{i}
$$

this equation becomes

$$
\frac{\partial \xi_{i} \rho}{\partial t}+\frac{\partial \xi_{i} \rho u}{\partial x}=S_{i}-\frac{\partial J_{i}}{\partial x}
$$

Note that at this point, no approximations have been made yet. Now we make the approximation; the diffusive flux is approximated using Fick's first law:

$$
J_{i} \approx-\rho D_{i} \frac{\partial \xi_{i}}{\partial x} .
$$


However, it is important to note that now, summing Equation (51) gives

$$
\frac{\partial \rho}{\partial t}+\frac{\partial \rho u}{\partial x}=\sum_{i=1}^{N_{\text {species }}} S_{i}-\sum_{i=1}^{N_{\text {species }}} \frac{\partial J_{i}}{\partial x}
$$

which is only equivalent to the single-vapor equations if the sum of the diffusive fluxes is zero:

$$
\sum_{i=1}^{N_{\text {species }}} \frac{\partial J_{i}}{\partial x}=0
$$

Taking the gradient of Equation (52) and using the partition-of-unity property of the mass fractions, one can see that one trivial solution for this condition is that all species have the same diffusion coefficient. Otherwise, if one assumes this condition is met and thus uses Equation (47), along with Equation (51) for all species excluding species $i$, then the following is required to ensure consistency:

$$
\frac{\partial J_{i}}{\partial x}=-\sum_{j \neq i}^{N_{\text {species }}} \frac{\partial J_{j}}{\partial x} .
$$

Bringing the sum inside the derivative on the right side and then integrating (which does make the condition more strict but is still useful for this analysis) gives the following definition for the diffusion coefficient for species $i$ :

$$
D_{i}=\frac{-\sum_{j \neq i}^{N_{\text {species }}} D_{j} \frac{\partial \xi_{j}}{\partial x}}{\frac{\partial \xi_{i}}{\partial x}} .
$$

Note that if the vapor mixture equation is used along with $N_{\text {species }}-1$ species equations, with Equation (54) assumed to be satisfied, then one does not actually need to use Equation (56); it is used implicitly. For a binary mixture $\left(N_{\text {species }}=2\right)$, this simply reduces to $D_{1}=D_{2}$. 


\section{Vapor Mixture Equations}

Recall the vapor equations for the seven-equation model, without any NCGs:

$$
\begin{gathered}
\frac{\partial \alpha_{v} \rho_{v} A}{\partial t}+\frac{\partial \alpha_{v} \rho_{v} u_{v} A}{\partial x}=S_{v} A \\
\frac{\partial \alpha_{v} \rho_{v} u_{v} A}{\partial t}+\frac{\partial \alpha_{v}\left(\rho_{v} u_{v}^{2}+p_{v}\right) A}{\partial x}=p_{\text {int }} A \frac{\partial \alpha_{v}}{\partial x}+p_{v} \alpha_{v} \frac{\partial A}{\partial x}+F_{v} A \\
\frac{\partial \alpha_{v} \rho_{v} E_{v} A}{\partial t}+\frac{\partial \alpha_{v} u_{v}\left(\rho_{v} E_{v}+p_{v}\right) A}{\partial x}=p_{\text {int }} u_{\text {int }} A \frac{\partial \alpha_{v}}{\partial x}+Q_{v} A,
\end{gathered}
$$

where the source terms are the following:

$$
\begin{aligned}
& S_{v} A \equiv S_{v}^{\text {int }} A+S_{v}^{\text {wall }} A, \\
& S_{v}^{\mathrm{int}} A \equiv \Gamma_{\ell \rightarrow v}^{\mathrm{int}} a_{\mathrm{int}} A, \\
& S_{v}^{\text {wall }} A \equiv \Gamma_{\ell \rightarrow v}^{\text {wall }} P_{\mathrm{hf}} \text {, } \\
& F_{v} A \equiv \lambda\left(u_{\ell}-u_{v}\right) A-F_{\text {wall }, v}^{\text {friction }} A-F_{\text {int }, \ell \rightarrow v}^{\text {friction }} A \\
& +\alpha_{v} \rho_{v} A g_{x}+\left(S_{v}^{\text {int }}+S_{v}^{\text {wall }}\right) u_{\text {int }} A, \\
& Q_{v} A \equiv \bar{p}_{\text {int }} \mu\left(p_{\ell}-p_{v}\right) A+\bar{u}_{\text {int }} \lambda\left(u_{\ell}-u_{v}\right) A \\
& -F_{\text {wall }, v}^{\text {friction }} u_{v} A-F_{\text {int }, \ell \rightarrow v}^{\text {friction }} u_{\text {int }} A \\
& +\alpha_{v} \rho_{v} u_{v} A g_{x}+Q_{v}^{\text {int }} a_{\mathrm{int}} A+\left(q_{v}^{\text {wall }}+q_{\ell}^{\text {wall, boil }}\right) P_{\mathrm{hf}} \\
& +S_{v}^{\text {int }}\left(H_{v}^{\text {int }}-\frac{p_{\text {int }}}{\rho_{\text {int }}}\right) A+S_{v}^{\text {wall }} H_{\ell} A .
\end{aligned}
$$

Now the presence of NCGs is considered. Assuming that there is no volumetric source of non-condensable gases, i.e., $S_{i}=0$ for $i=1, \ldots, N_{\mathrm{NCG}}$, the vapor mixture equation is simply the following:

$$
\frac{\partial \alpha_{v} \rho_{v} A}{\partial t}+\frac{\partial \alpha_{v} \rho_{v} u_{v} A}{\partial x}=S_{v, 0} A
$$

This equation replaces the single vapor equation, given by Equation (57). With the addition of the NCGs, there are $N_{\mathrm{NCG}}$ new unknowns in the system: $\xi_{v, i}$ for $i=1, \ldots, N_{\mathrm{NCG}}$ 
and thus $N_{\mathrm{NCG}}$ new equations are required. The associated equations will be the species equations for each NCG:

$$
\frac{\partial \alpha_{v} \xi_{v, i} \rho_{v} A}{\partial t}+\frac{\partial \alpha_{v} \xi_{v, i} \rho_{v} u_{v} A}{\partial x}=-\frac{\partial J_{v, i}}{\partial x}
$$

where the diffusive flux is

$$
J_{v, i}=-\alpha_{v} \rho_{v} D_{v, i} \frac{\partial \xi_{v, i}}{\partial x} A
$$

\subsection{Spatial Discretization and Boundary Conditions}

This section describes how Equation (66) is spatially discretized. Note that Equation (65) appears in the PDE system as well, but it looks the same as its no-NCG version, so it does not need discussion.

The time derivative, advection, and volumetric source terms of Equation (66) are discretized just as the corresponding terms in the vapor mixture mass equation, so the only term that needs to be discussed is the the new term, containing the diffusion flux. For the continuous Galerkin finite element, the term is integrated by parts:

$$
\left(\frac{\partial J_{v, m}}{\partial x}, \varphi_{i}\right)_{\Omega}=-\left(J_{v, m}, \frac{\partial \varphi_{i}}{\partial x}\right)_{\Omega}+\left\langle J_{v, m}, \varphi_{i} n_{x}\right\rangle_{\Gamma} .
$$

The boundary term is usually evaluated according to boundary conditions, but here an approximation is made to avoid these complications: the diffusive flux at the boundary is assumed to be zero. Thus,

$$
\left(\frac{\partial J_{v, m}}{\partial x}, \varphi_{i}\right)_{\Omega}=-\left(J_{v, m}, \frac{\partial \varphi_{i}}{\partial x}\right)_{\Omega} .
$$

Note that this is not an approximation for wall boundary conditions, only for inlets and outlets.

For finite volume discretization, the diffusion term is integrated over the volume, leading to the following:

$$
\int_{V_{i}} \frac{\partial J_{v, m}}{\partial x} \mathrm{~d} V=J_{v, m, i+1 / 2}-J_{v, m, i-1 / 2}
$$

where again, the diffusive flux is assumed to be zero if it corresponds to a boundary edge. 


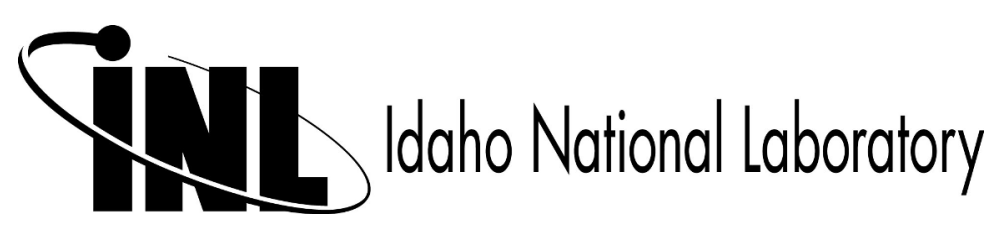

